\title{
DOE/PC/90/80--T/3
}

QUARTERLY REPORT

$10 / 01 / 94$ to $1 / 30 / 95$

\section{NUMERICAL STUDY OF THE FLOW OF GRANULAR MATERIALS DOWN AN INCLINED PLANE}

\author{
Project Director: $\quad$ K.R. Rajagopal \\ Department of Mechanical Engineering \\ University of Pittsburgh \\ Pittsburgh, PA 15261
}

Contract No: DE-AC22-91PC90180

Contract Period: 12/13/1990 - 12/13/1995

\section{DISCLAIMER}

\begin{abstract}
This report was prepared as an account of work sponsored by an agency of the United States Government. Neither the United States Government nor any agency thereof, nor any of their employees, makes any warranty, express or implied, or assumes any legal liability or responsibility for the accuracy, completeness, or usefulness of any information, apparatus, product, or process disclosed, or represents that its use would not infringe privately owned rights. Reference herein to any specific commercial product, process, or service by trade name, trademark, manufacturer, or otherwise does not necessarily constitute or imply its endorsement, recommendation, or favoring by the United States Government or any agency thereof. The views and opinions of authors expressed herein do not necessarily state or reflect those of the United States Government or any agency thereof.
\end{abstract}




\section{DISCLAIMER}

Portions of this document may be illegible in electronic image products. Images are produced from the best available original document. 


\section{SUMMARY}

In the previous report the governing equations were derived for the flow of granular materials down an inclined plane using the kinetic theory based model proposed by Boyle and Massoudi (1990). For a fully developed flow of these materials down an inclined plane, the equations reduce to a system of coupled non-linear ordinary differential equations. The resulting boundary value problem was solved numerically and a parametric study was carried out to delineate how the various non-dimensional parameters affect the structure of the solution. Further parametric studies were carried out and the results were in keeping with the previous studies. Also, a study of the flow of granular material between two vertical plates was carried out and compared with experiments in order to evaluate the continuum model. 


\section{INTRODUCTION}

Understanding the behavior of flowing granular materials is motivated by the need to determine bin and hopper geometries that permit granular solids to flow freely, to devise reasonable methods for

predicting the stresses that develop on the walls of the container and to characterize scale-up factors for solids transport.

Granular materials exhibit phenomena, such as normal stress differences in simple shear flow which is typical of materials whose response in non-linear. For instance, simple shearing motion is not determined by the shear force alone but also by the normal forces that manifest themselves due to the shearing. Reynolds [5] observed that for a shearing motion to occur in a bed of closely packed particles, the bed must expand to increase the volume of its voids. He termed this phenomenon "dilatancy." Many of the existing theories use this observation to relate the applied stress to the voidage and the velocity. Also, experiments on granular materials indicate the presence of normal stress effects (cf. Savage [4], Baganold [6]).

Granular materials are unlike solids in that they conform to the shape of the vessel containing them, thereby exhibiting fluid like characteristics. On the other hand, they cannot be considered as fluids, as they can be heaped. The characteristics of the individual particles that constitute the bulk and the interstitial dynamics determine the behavior both at rest and during flow. Due to their complexity, the modeling of granular materials would require a fusion of the ideas from both solid and fluid mechanics. 
Several models have been proposed, on the basis of kinetic theory and continuum mechanics, to describe the behavior of granular materials (cf. Lun et al. [7], Jenkins and Savage [8], Ahmadi and Shahinpoor [9], Boyle and Massoudi [10], Goodman and Cowin [1,2], Savage [4] and Rajagopal and Massoudi [3]). Some of these models have been proposed for loosely packed materials, while others have been proposed for rapidly flowing materials, and still others for different kinematical situations. The recent review article by Hutter and Rajagopal [12] discusses the status of the numerous models that abound in the literature and delineate the domain of applicability. In this work, we use the continuum model used earlier by Rajagopal and Massoudi [3] to study flow of granular materials down a vertical channel.

It is appropriate at this juncture to point out that most of the models that stem from kinetic theory are subsumed by the model due to Boyle and Massoudi [10] based on an approach similar to the Enskog dense gas theory. This model predicts the presence of normal stress difference in simple shear flow. Interestingly, the constitutive expression for the stress can be put into the form of the model considered in this paper, with the material moduli having specific representation. We shall not get into a lengthy discussion of the status of this model but refer the reader to [10] for the same. Suffice to say that the model has been found to predict qualitatively many of the features observed in the flow of granular materials (cf. Rajagopal, Troy and Massoudi [11]). While other models might also predict such features, they have not usually been derived within the context of the 
mechanics of non-linear materials, while also being consistent with models that arise out of a kinetic theory approach.

\section{Governing Equations}

We shall now proceed to derive the governing equations for the flow of granular materials down a vertical channel (cf. Figures 1). We assume that the cauchy stress $\boldsymbol{T}$ for the granular materials is given by (cf. Goodman \& Cowin [1,2]. Rajagopal \& Massoudi [3]):

$$
T=\left\{\beta_{0}(v)+\beta_{1}(v) \nabla v+\beta_{2}(v) \operatorname{tr} D\right\} 1+\beta_{3}(v) D+\beta_{4}(v) \nabla v \otimes \nabla v
$$

where $v$ denotes the volume fraction of the particles, $\mathbf{D}$ the stretching tensor associated with the motion of particles.

In general, if we were to start with a stress $\mathbf{T}$ that depends on $v, \nabla v$ and $\mathbf{D}$, we would obtain a model that is more general than that given by (1) wherein the material moduli depend on $v$ and the invariants of $\mathbf{D}, \nabla v \otimes \nabla v, \mathbf{D} \nabla v \otimes \nabla v+\nabla v \otimes \mathbf{D} \nabla v$. Such constitutive representations can account for cohesion or otherwise, depending on the specific forms of the material functions, i.e. $\beta^{s}$. Here, $\beta_{0}(v)$ is similar to pressure in a compressible fluid and is given by an equation of state, $\beta_{2}(v)$ is similar to the second coefficient of viscosity in a compressible fluid, $\beta_{1}(v)$ and $\beta_{4}(v)$ are material parameters that reflect the distribution of the granular materials and $\beta_{3}(v)$ is the viscosity of the granular materials. In general, the material properties $\beta_{0}$ through $\beta_{4}$ are functions of the volume fraction, temperature, and the principal invariants of the stretching tensor $\mathbf{D}$, given by

$$
D=\frac{1}{2}\left[(\nabla u)+(\nabla u)^{T}\right]
$$


where $\mathbf{u}$ is the velocity of the particles. In equation (1), $\mathbf{1}$ is the identity tensor, $\nabla$ the gradient operation, $\otimes$ indicates the dyadic (outer product) of two vector and tr denotes the trace of a tensor. The volume fraction $(v)$ is related to the bulk density $(\rho)$ through the following relation

$$
\rho=\gamma
$$

where $\gamma$ is the particle or grain density at the place $x$ and at time $t$.

Rajagopal, Troy and Massoudi [11] have studied the question of existence of solutions of the flow of a granular material modeled by (1) flowing down an inclined plane and prove that non-unique solutions are possible. These solutions are in keeping with the experimental results of Ahn [13].

Following Rajagopal and Massoudi [3], we assume that the material parameters have the following structure

$$
\begin{aligned}
& \beta_{0}(v)=k v \\
& \beta_{1}(v)=\beta_{10}+\beta_{11} v+\beta_{12} v^{2} \\
& \beta_{2}(v)=\beta_{20}+\beta_{21} v+\beta_{22} v^{2} \\
& \beta_{3}(v)=\beta_{30}+\beta_{31} v+\beta_{32} v^{2} \\
& \beta_{4}(v)=\beta_{40}+\beta_{41} v+\beta_{42} v^{2}
\end{aligned}
$$

The above representation can be viewed as a Taylor series approximation for the material properties. Such a quadratic dependence, at least for the viscosity $\beta_{3}$ is based on the dynamic simulations of particle interactions by Walton and Braun [14]. Further restrictions on the coefficients can be obtained by using the argument that as $v \rightarrow 0$, the stress $\mathbf{T}$ should vanish, accordingly: 


$$
\beta_{30}=\beta_{20}=0
$$

A rationale for the structure of $\beta$ 's can be found in Rajagopal and Massoudi [3]. Furthermore, Rajagopal et al. [3] have shown that

$$
k<0
$$

as compaction should lead to densification of the material. In general the second law of thermodynamics would also place restrictions on the nature of the material parameters. This issue is outside the scope of this work.

The governing equations are the equations for the conservation of mass and momentum:

$$
\frac{\partial \rho}{\partial t}+\operatorname{div}(\rho u)=0
$$

and

$$
\rho \frac{d u}{d t}=d i v T+\rho b
$$

Here, $\frac{\partial}{\partial t}$ is the partial derivative with respect to time, $\frac{d}{d t}$, is the material time derivative and $\mathbf{b}$ is the body force.

For the case of the flow of granular materials down a vertical channel, we assume that the motion is steady, the granular particles are incompressible, $(\gamma=$ constant $)$, and that the constitutive expression for the stress tensor is given by (1). In general, rapid flows in vertical channels will not necessarily be fully developed. However, if the channel is long enough and the feed rate not too large, the assumption of a fully developed flow might be reasonable. In the experiments of Savage [4], he was able to realize fully developed flow between vertical channels and hence in our study here, we shall seek 
such flows. We shall suppose that the density and velocity fields are of the form

$$
\begin{aligned}
& v=v(y) \\
& u=u(y) i
\end{aligned}
$$

respectively.

Let us consider the vertical channel shown in Figure 1. The two vertical walls are separated by a distance $2 \mathrm{~h}$. In order to solve the problem, we consider two cases:

Case 1: $\quad \beta_{1}, \beta_{2}$, and $\beta_{4}$ are constants

Case 2: $\quad \beta_{1}, \beta_{2}$ and $\beta_{4}$ vary quadriatically in volume fraction In both Case 1 and Case 2, $\beta_{0}$ and $\beta_{3}$ are assumed to be of the form

$$
\beta_{0}=k v
$$

and

$$
\beta_{3}=\hat{\beta}_{3}\left(v+v^{2}\right)
$$

The rationale for the more general choice is provided in Rajagopal and Massoudi [3].

Case 1 can be solved analytically, but Case 2 has to be solved numerically. We shall consider each case separately.

Case 1: $\quad \beta_{1}, \beta_{2}$, and $\beta_{4}$ are constants

In this case, the conservation of mass is identically satisfied and the conservation of linear momentum reduces to

$$
\begin{aligned}
& k \frac{d v}{d y}+2 \beta_{1}\left(\frac{d v}{d y}\right)\left(\frac{d^{2} v}{d y^{2}}\right)+2 \beta_{4}\left(\frac{d v}{d y}\right)\left(\frac{d^{2} v}{d y^{2}}\right)=0 \\
& \frac{\hat{\beta}_{3}(1+2 v)}{2} \frac{d u}{d y}\left(\frac{d v}{d y}\right)+\frac{\hat{\beta}_{3}}{2}\left(v+v^{2}\right) \frac{d^{2} u}{d y^{2}}+\rho g=0
\end{aligned}
$$


We non-dimensionalize equations, (13) and (14), by defining $\bar{y}=\frac{y}{h}$ and $\bar{u}=\frac{u}{U_{0}}$; where $U_{0}$ is the maximum velocity. We get

$$
R_{2}\left(\frac{d v}{d y}\right) \frac{d^{2} v}{d \bar{y}^{2}}+R_{1} \frac{d v}{d \bar{y}}=0
$$

and

$$
\frac{d^{2} \bar{u}}{d \bar{y}^{2}}+\frac{(1+2 v)}{v(1+v)}\left(\frac{d v}{d \bar{y}}\right) \frac{d \bar{u}}{d \bar{y}}+\frac{1}{R_{3}(1+v)}=0
$$

In equations (15) and (16), $R_{1}, R_{2}$ and $R_{3}$ are dimensionless quantities given by

$$
R_{1}=\frac{k}{\gamma g h} ; R_{2}=\frac{2\left(\beta_{1}+\beta_{4}\right)}{h^{3} \gamma g} ; R_{3}=\frac{\hat{\beta}_{3} U_{0}}{2 \gamma g h^{2}}
$$

The parameters $R_{1}, R_{2}$ and $R_{3}$ have the following physical interpretations: $R_{1}$ is the ratio of the pressure force to the gravity force, $R_{2}$ is the ratio of the forces developed in the material due to the distribution of voids to the force of gravity, and $R_{3}$ is the ratio of the viscous force to the force of gravity.

Notice, that (15) is an ordinary differential equation in $v$, while (16) is an ordinary differential equation in both $v$ and $\bar{u}$.

$$
\begin{array}{lll}
\bar{u}=0 & \text { at } & \bar{y}= \pm 1 \\
\frac{d v}{d \bar{y}}=0 & \text { at } & \bar{y}=0 \\
Q=\frac{N}{h}=\int_{-1}^{1} v d \bar{y} ; & \text { ( } Q \text { being given) }
\end{array}
$$


Equation (18) 1 is the adherence boundary condition for the nondimensional velocity and (18) 2 is a consequence of mid-planar symmetry, and (19) indicates that the amount of material flow $(Q)$ through the channel is a constant. From equation (15) we get

$$
v=\frac{Q}{2}=\text { constant }
$$

or

$$
v=-\frac{R_{1}}{2 R_{2}} \bar{y}^{2}+\frac{R_{1}}{6 R_{2}}+\frac{Q}{2}
$$

Equation (20) indicates that the volume fraction through the channel is a constant. As, this condition is not realized in real flows, the solution is degarded. Substituting (21) into (16) and using the appropriate conditions we get

$$
\begin{aligned}
& \bar{v}=\frac{1}{15 B R_{3}}\left\{(-1.667 B+10 A-5) \ln \left[\frac{5000 B \bar{y}^{2}-1667 B+10,000 A+10,000}{3333 B+10,000 A+10,000}\right]-(10 A-1.667 B)\right. \\
& \left.\ln \left[\frac{5000 B \bar{y}^{2}-1667 B+10000 A}{3333 B+10000 A}\right]\right\}
\end{aligned}
$$

$$
\begin{array}{ll}
\text { where } & A=Q / 2 \\
\text { and } & B=-\frac{R_{1}}{R_{2}}
\end{array}
$$

Case 2: $\quad \beta_{1}, \beta_{2}, \beta_{4}$ are quadratic functions of $v$

We assume that material parameters $\beta$ 's are quadratic function of $v$ and for the sake of convenience and simplicity, choose

$$
\begin{aligned}
& \hat{\beta}_{1}=\beta_{10}=\beta_{11}=\beta_{12} \\
& \hat{\beta}_{2}=\beta_{21}=\beta_{22}
\end{aligned}
$$




$$
\hat{\beta}_{4}=\beta_{40}=\beta_{41}=\beta_{42}
$$

Thus, the material parameters may be written as

$$
\begin{aligned}
& \beta_{0}(v)=k v \\
& \beta_{1}(v)=\hat{\beta}_{1}\left(1+v+v^{2}\right) \\
& \beta_{2}(v)=\hat{\beta}_{2}\left(v+v^{2}\right) \\
& \beta_{3}(v)=\hat{\beta}_{3}\left(v+v^{2}\right) \\
& \beta_{4}(v)=\hat{\beta}_{4}\left(1+v+v^{2}\right)
\end{aligned}
$$

Once again, by virtue of (9) and (10), the conservation of mass is identically satisfied. The balance of linear momentum, after appropriate non-dimensionlization yields:

$$
\frac{d v}{d \bar{y}}+\frac{S_{2}}{R_{1}}\left(1+v+v^{2}\right)\left(\frac{d v}{d \bar{y}}\right) \frac{d^{2} v}{d \bar{y}^{2}}+\frac{S_{2}(1+2 v)}{2 R_{1}}\left(\frac{d v}{d \bar{y}}\right)^{3}=0
$$

and

$$
\frac{d^{2} \bar{u}}{d \bar{y}^{2}}+\frac{(1+2 v)}{v(1+v)}\left(\frac{d v}{d \bar{y}}\right)\left(\frac{d \bar{u}}{d \bar{y}}\right)+\frac{1}{R_{3}(1+v)}=0,
$$

where $\bar{y}=\frac{y}{h}$ and $\bar{u}=\frac{u}{U_{0}}, R_{3}$ and $R_{1}$ are given by (17), while $S_{2}$ is given by (29) which has the same physical interpretation as $R_{2}$. Notice, equation (26) is identical to (16) but (25) has an extra term when compared to (15) because of the dependence of all the material parameters on the volume fraction $v$. Equation (25) admits the trivial solution of $v=$ constant, and once again we ignore it on the basis of experimental observations. Equations (25) and (26) subject to (18) and (19) are solved numerically using COLSYS (cf. Ascher et al. [15])

Here, we also consider the case of slip at the wall, by assuming that the slip velocity is proportional to the stress vector at the wall given by 


$$
u_{s}=\hat{f}\left((T n)_{x},(T n)_{y}\right)
$$

where $\boldsymbol{T}$ is the stress tensor, $\boldsymbol{n}$ is a unit normal vector, and $\hat{f}$ in general could be a function of surface roughness, volume fraction, shear rate, etc. (cf. Pearson and Petrie [16], Craig et al. [17], Hanes and Inman [18], Hutter et al. [19, 20] and Schowalter [21]). With the constitutive equation (1), the slip boundary condition in dimensionless form will be

$$
\bar{u}=f\left[R_{1} v+\frac{S_{2}}{2}\left(1+v+v^{2}\right)\left(\frac{d v}{d y}\right)^{2}+R_{3}\left(v+v^{2}\right) \frac{d \bar{u}}{d \bar{y}}\right]
$$

The dimensionless parameters $f$ and $S_{2}$ are given by

$$
f=\frac{\hat{f h} \gamma g}{U_{0}} ; S_{2}=\frac{2\left(\hat{\beta}_{1}+\hat{\beta}_{4}\right)}{h^{3} \gamma g}
$$

\section{Results and Discussions}

We now solve equations (25) and (26) subject to the appropriate boundary conditions numerically and compare the results with the experimental data of Savage [4].

We solved both the cases for slip and no-slip using a collocation code COLSYS (cf. Ascher et al [15]). COLSYS is implemented using a B-spline basis function. COLSYS features an adaptive mesh selection procedure based on error estimation. The mesh points are repositioned to roughly equidistribute the error, which is estimated using mesh halving and checked using prescribed tolerances. The integral condition for the volume fraction is implemented using a secant shooting method to refine initial guesses for the value of volume fraction prescribed. 
Figure 2 shows a comparison of the experimental data of Savage [4] with the numerical predictions in this work, and Figure 3 is the corresponding volume fraction profile. It should be emphasized that we used all the physical data such as the density of the solid, etc., from the experimental results of Savage. However, the non-dimensional parameters, $R_{1}, R_{2}$ and $R_{3}$ all involve material moduli that appear in [4] for which experimental values are at this moment unavailable. It is observed that the experimental results of Savage [4] and our numerical results agree well with each other, for the values shown. It is however possible that these values may not indeed be the real values for the material in question. Thus, we also provide a parametric study of the governing equations. Such a parametric study can be used in conjunction with carefully carried out experiments in channel flows to either validate or render invalid the constitutive model that is proposed here. In that sense the parametric study carried out here could be of some use.

Figures 4 to 15 show the effect of the various non-dimensional parameters and the boundary conditions on the velocity and volume fraction distribution in the vertical channel. Such an analysis is just a parametric study as we do not have experimental data, for such values of the parameters, to compare with. However, they do provide results that can be compared against future experimental results. The equations of mass and momentum, (15) and (16) for the case where $\beta_{1}, \beta_{2}$, and $\beta_{4}$ are constants are solved subject to the conditions (18) and (19). Figures $4 \& 5$ show the effect of decreasing the value of $R_{1}$, while keeping $R_{2}, R_{3}$ and $Q$ the same, on the volume fraction and the velocity in the channel under no slip conditions. It is observed 
from Figure 4 that irrespective of the value of $R_{1}$, the volume fraction is maximum at the boundaries and minimum at the center of the channel. The change in volume fraction is maximum at the boundaries and minimum at the center of the channel. The change in volume fraction in the channel increases as the value of $R_{1}$ decreases (i.e., becomes more negative.) $R_{1}$ is the ratio of the pressure force to the gravity force, decrease in $R_{1}$ implies an increase in the compressive pressure, which causes the granular material to redistribute as would be expected. Decrease in $R_{1}$, decreases the velocities as shown in Figure 5. Figures 6 and 7 represent the plots of volume fraction and velocity in a vertical channel where $\beta_{1}, \beta_{2}$ and $\beta_{4}$ are taken as quadratic functions of the volume fraction $v$. Figures 6 and 7 show the effect of decrease in $R_{1}$, and the results are very similar to Figures 4 and 5 respectively, the difference being the magnitudes of the values of the volume fraction and velocity. Since we are only interested in the qualitative nature of the plots and due to the similar characteristics of the plots in both cases ( $\beta$ 's constant and $\beta$ 's quadratic functions of $v$ ), only the plots for $\beta$ 's as quadratic functions of $v$ will be presented here.

In Figures 4 to 7 the value of $R_{2}$ and $S_{2}$ are taken as positive. Figures 8 and 9 show the effect of decreasing $R_{1}$ with $S_{2}$ negative. It is noticed that the volume fraction profiles are exactly the opposite of the earlier plots. In this context we refer to the theoretical results in $[22,23]$ and the experimental results in [4], where similar features are observed. In Figure 8, the plot of volume fraction in the channel is maximum at the center of the channel and minimum at the edges. Also decreasing $R_{1}$ increases the volume fraction at the center of the 
channel and decreases the volume fraction at the boundary. In Figure 9, we see that decreasing $R_{1}$ increases the velocity of the granular material flowing in the channel. We can also notice that the velocity profiles are not parabolic but seem to suggest plug flow i.e., the variation in velocity in the central region of the pipe is much smaller than that along the edges. This result is to be expected, as the amount of material (Q) flowing through the channel is a constant. Thus, a decrease in the core of the channel will be compensated by a corresponding increase at the edges. As $S_{2}$ is ratio of the forces developed in the material due to the distribution of voids to the force of gravity, a decrease in $S_{2}$ would imply a decrease in the forces developed in the material due to the distribution of voids which would in turn cause a decrease in velocity. This behavior is illustrated in Figures 10 and 11.

Figure 12 shows the effect of an increase in $R_{3}$ on the velocity profile. Increasing the value of $R_{3}$ decreases the velocity. This is to be expected as $R_{3}$ is the ratio of the viscous forces to gravity, and thus increasing $R_{3}$ in effect increases the 'viscosity' of the material, all other conditions remaining the same. The governing equations for the volume fractions (15) and (25) do not contain $R_{3}$. Hence any change in $R_{3}$ does not affect the volume fraction in the channel. Figure 14 shows the effect of increasing the value of $Q$ on the velocity distribution in channel. As is to be expected, increasing the amount of material flowing through the channel decreases the dimensionless velocity in the channel and increases the volume fraction of the material flowing through the channel (c.f. Figure 13). Figure 15 illustrates the effect of slip and no-slip at the boundary. The 
dimensionless velocity for slip and no slip cases have similar profiles, but the magnitudes are markedly different. The dimensionless velocity at the center line is much greater when the material slips at the boundary.

The results discussed above agree qualitatively with the work of Nunziato et al. [22], who used a non-linear version of the Goodman and Cowin $[1,2]$ to study the gravity flow in a closed vertical channel. Their predictions for the volume fraction and velocity profiles agree qualitatively with our results. Johnson et al. [23] used mixture theory to study the flow of granular materials between horizontal parallel plates found in the volume fraction profiles that are similar to ours.

\section{Acknowledgement}

K. R. Rajagopal and R. Gudhe thank the Pittsburgh Energy Technology Center, U. S. Department of Energy for its support. 


\section{NOMENCLATURE}

\begin{tabular}{cl} 
Symbol & Explanation \\
\hline D & Stretching tensor \\
$\mathbf{T}$ & Cauchy stress tensor \\
$\mathbf{b}$ & Body force \\
$\mathrm{g}$ & Acceleration due to gravity \\
$2 \mathrm{~h}$ & Distance between the two vertical walls \\
$\mathbf{n}$ & Unit normal vector \\
$\mathbf{u}$ & Velocity of the particles \\
$\beta_{\mathrm{i}}$ & Material parameters (i $=0-4)$ \\
$\gamma$ & Distributed mass density \\
$v$ & Volume fraction \\
$\mathrm{p}$ & Bulk mass density
\end{tabular}




\section{REFERENCES}

1. M. A. Goodman, and S. C. Cowin: Two Problems in the Gravity Flow of Granular Materials. J. Fluid Mechanics, 45, (1971), 32 1-339.

2. M. A. Goodman, and S. C. Cowin: A Continuum Theory for Granular Materials. Arch. Rat. Mech and Aral 44, (1972), 249-266.

3. K. R. Rajagopal, and M. Massoudi: A Method for Measuring Material Modull of Granular Materlals: Flow in an orthogonal Rheometer. (1990), DOE/PETC/TR-90/3.

4. S. B. Savage: Gravity Flow of Cohesionless Granular Materiais in Chutes and Channels. J. Fuid Mechanics, 93. (1979), 53-96.

5. O. Reynolds: Experiments Showing Dilatancy a Property of Granular Material, Possibly Connected with Gravitation. Proc. Roy. Inst. of Gr. Britian. 11. (1886). 354-363.

6. R. A. Bagnold: Experiments on a Gravity Free Dispersion of Large Solid Spheres in a Newtonian Fluid. Proc. Roy. Soc. London, 225, (1956), 49.

7. C. K K Lun, S. B. Savage, D. J. Jeffrey and N. Chepurniy: Kinetic Theories for Granular Flow: Inelastic Particles in Couette Flow and Slightly Inelastic Particles in a General Flow Field. J. Fluid Mechanics, 140. (1984), 223-256.

8. J. T. Jenkins and S. B. Savage: A Theory for the Rapid Flow of Identical Smooth, Nearly Elastic, Spherical Particles. J. Fluid Mechanics, 130, (1983), 187-202.

9. G. Ahmadi and M. Shahinpoor: A Kinetic Model for Rapid Flows of Granular Materials. Int. J. of Non-Linear Mechanics, 19, (1984), 177186.

10. E. J. Boyle and M. Massoudi: A Theory for Granular Materials Exhibiting Normal Stress Effects based on Enskog's Dense Gas Theory. Int. J. Engng. Sci, 28, (1990), 1261-1275.

11. K. R. Rajagopal, W. C. Troy, and M. Massoudi: Existence of Solutions to the Equations Governing the Flow of Granular Materials. European J. Mech B/Fuids, 11, (1992), 265-276.

12. K. Hutter and K. R. Rajagopal: On Flows of Granular Materials: Review Article, Continuum Mechanics and Thermodynamics., Vol. 6 No. 2. (1994). 81-139.

13. H. Ahn: Experimental and Analytical Investigation of Granular Materials, Ph.D. Thesis, Cal. Tech. (1989).

14. O. R. Walton and R. L. Braun: Viscocity. Granular-Temperature , and Stress Calculations for Shearing Assemblies of Inelastic, Frictional Disks. J. Rheology. 30, (1986), 949-980.

15. V. Ascher, J. Christiansen, and R. D. Russel: Collocation Software for Boundary Value ODE's. ACM Transaction on Mathematical Software, 7/2. (June 1981), 209-22. 
16. J. R. A. Pearson and C. J. S. Petrie: On the Melt-Flow Instability of Extruded Polymers. Proc. of Fourth International Congress on Rheology. Brown Untversity, Ed. E. H. Lee (1965).

17. K. R. Craig. H. Buckholtz and G. Domoto: The Effects of Shear Surface Boundaries on Stresses for Rapid Shear of Dry Powders. ASME J. Tribology, 109. (1987), 232.

18. D. M. Hanes and D. I. Inman: Observations of Rapidly Flowing Granular Materials. J. Fuid Mechanics, 150, (1985), 357.

19. K Hutter, F. Szddarovsziky, and S. Yakowitz: Plane Steady Shear Flow of a Cohesionless Granular Material Down an Inclined Plane: A Model for Flow Avalanches Part-1: Theory. Acta Mech 63. (1986a). 87-112.

20. K. Hutter, F. Szddarovszky, and S.Yakowitz, Plane Steady Shear Flow of a Cohesionless Granular Material Down an Inclined Plane: A Model for Flow Avalanches Part-II: Theory. Acta Mech. 65. (1986b), 239-261.

21. W. R. Schowalter: The Behavior of Complex Fluids at Solld Boundaries. J. Non-newtonian Fluid Mechanics, 29, (1988), 25 .

22. J. W. Nunziato, S. L. Passman and J. P. Thomas, Jr.: Gravitational Flow of Granular Materials with Incompressible Grains. J. Rheology. 24. (1980), 395-420.

23. G. Johnson, M. Massoudi, and $\mathbf{K}$. R. Rajagopal: Flow of a Fluid-Solid Mixture Between Flat Plates. Chem Engr. Science,.46, (1991), 17131723. 


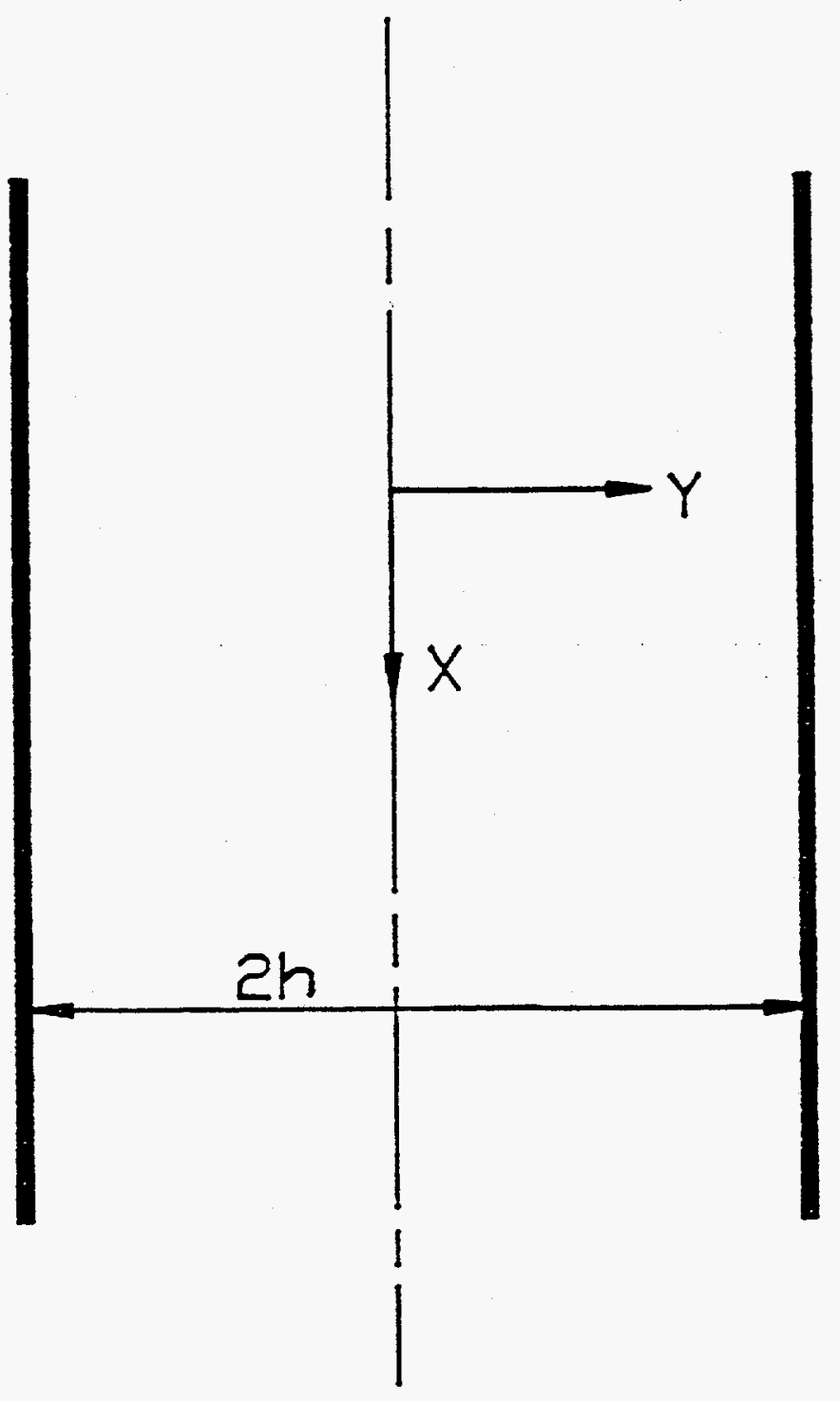

Figure 1. Flow Down a Vertical Channel. 


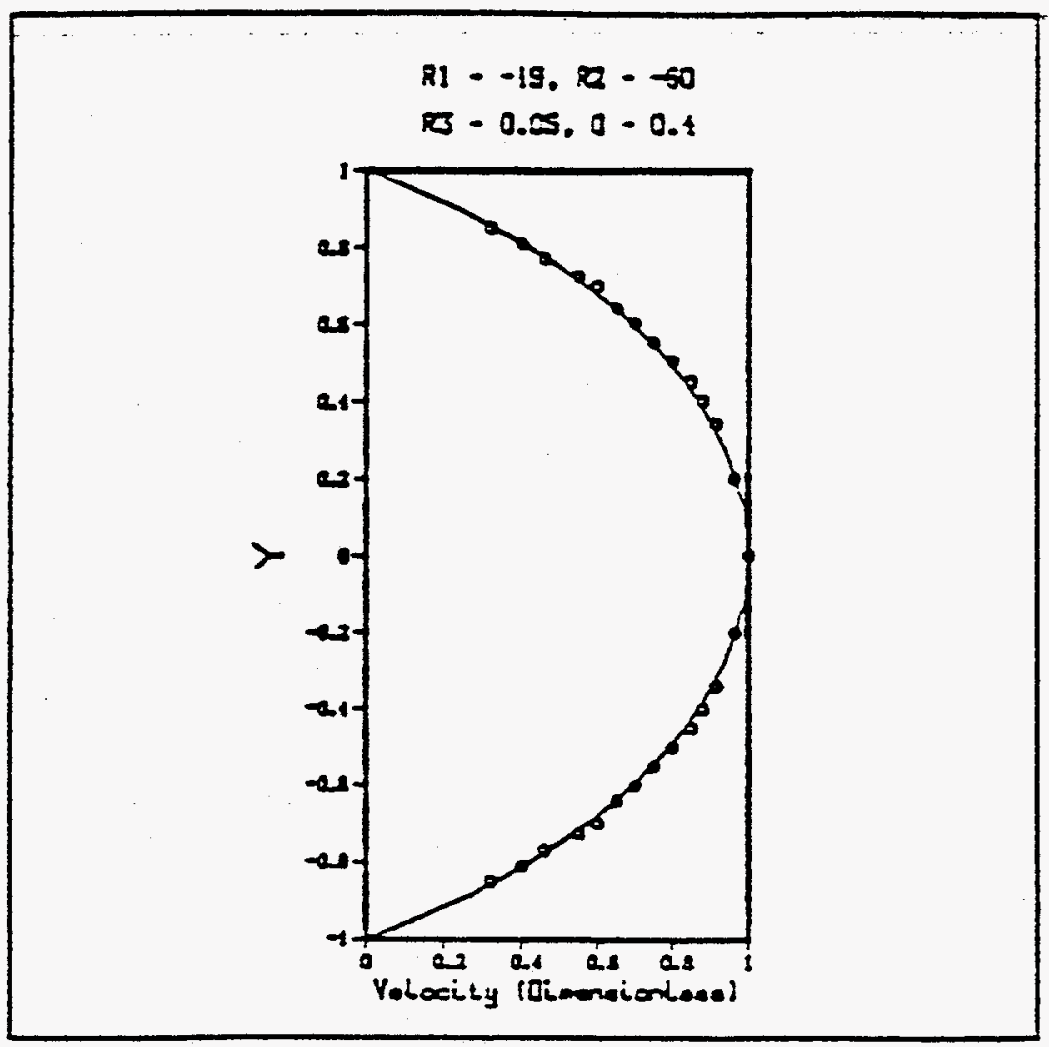

Figure 2. Comparision of Velocity Profile with the Experimental Data of Savage [4].

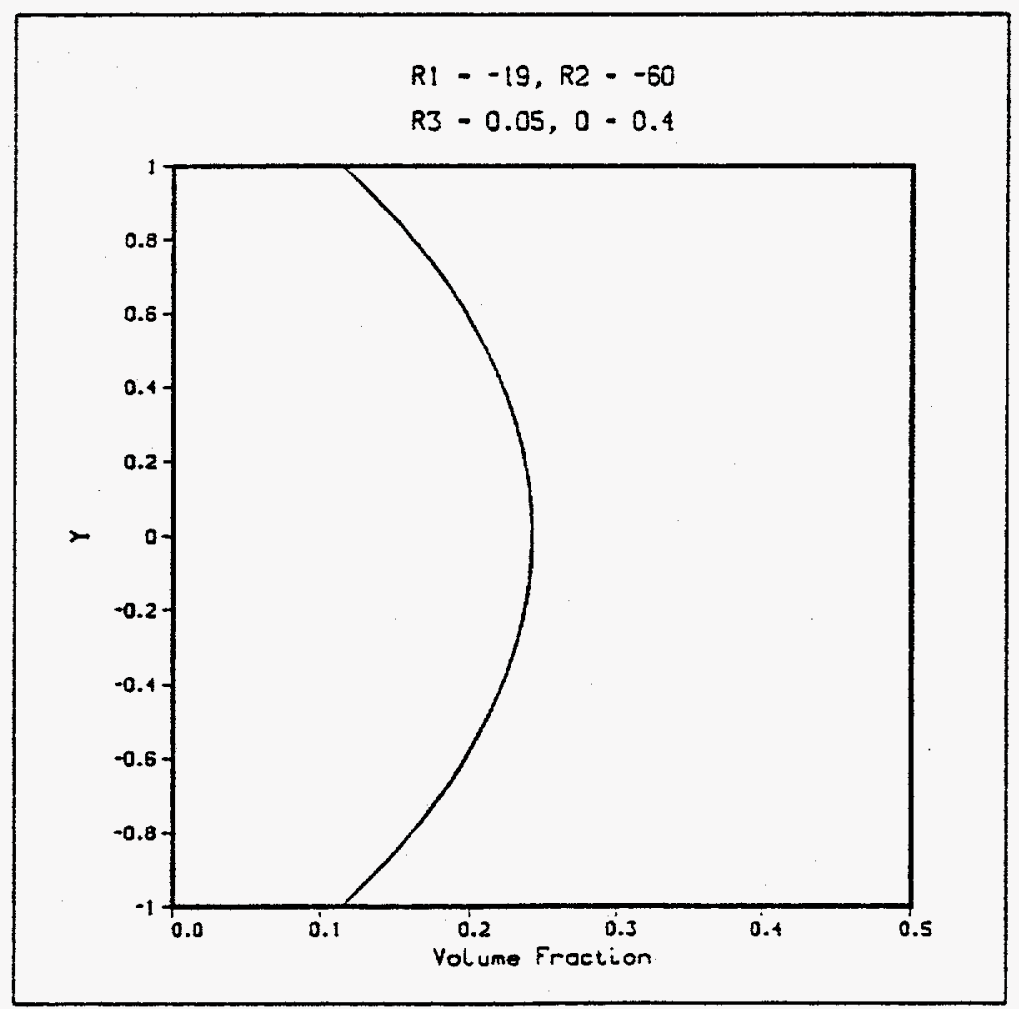

Figure 3. Volume Fraction Profile. 


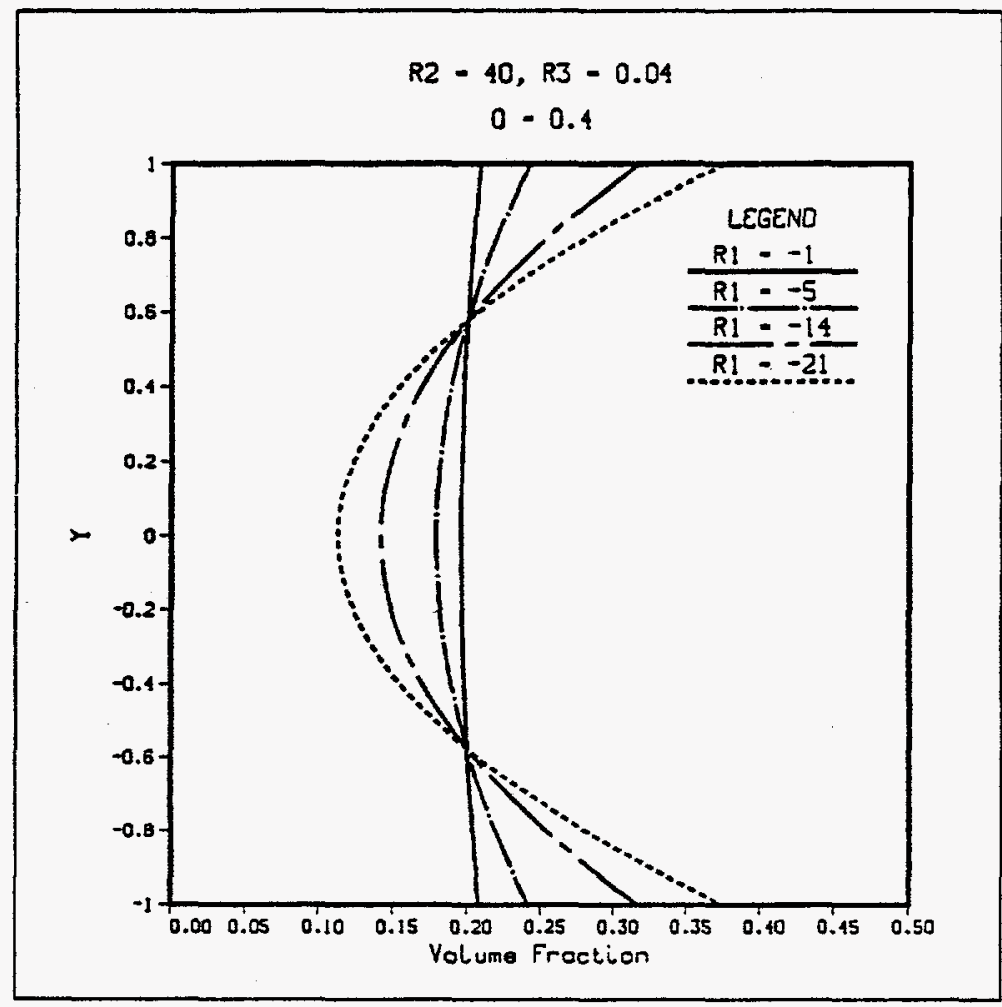

Figure 4. Effect of $R_{1}$ onVolume Fraction Profiles (Case 1).

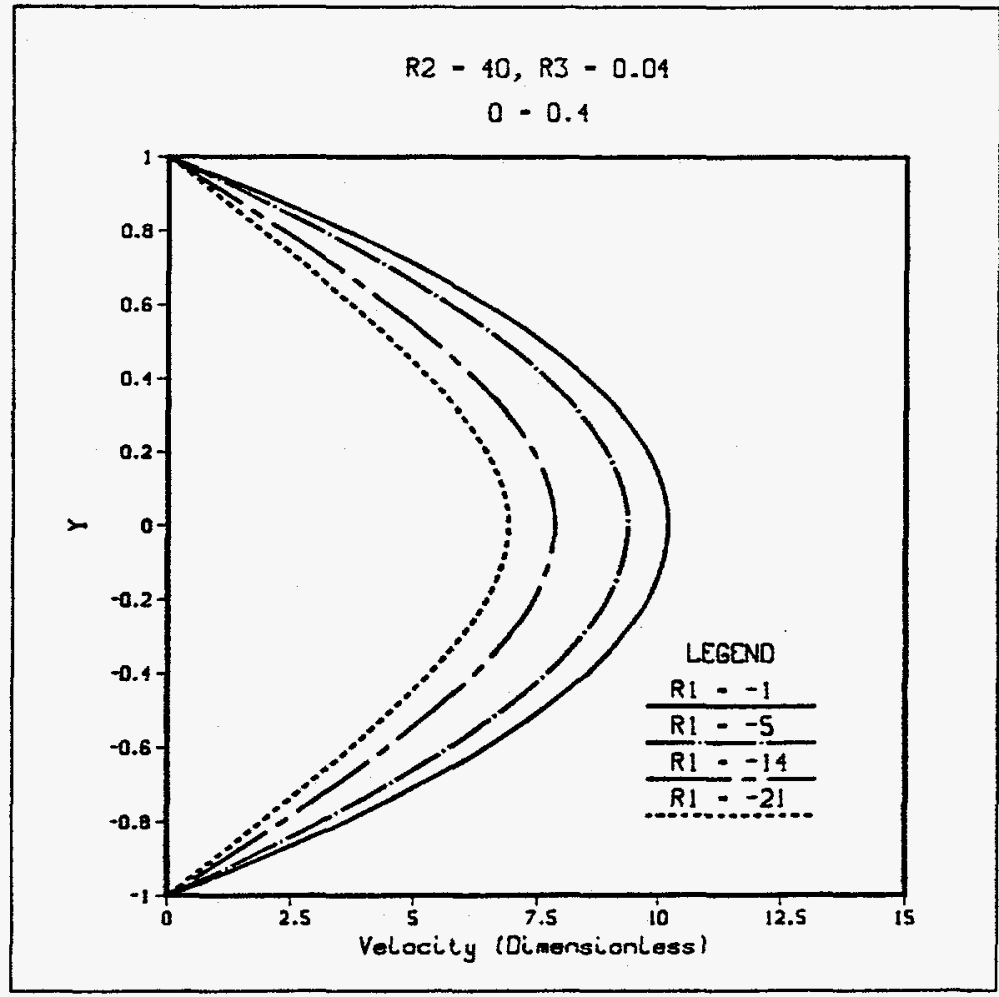

Figure 5. Effect of $R_{1}$ on Velocity Profiles (Case 1). 


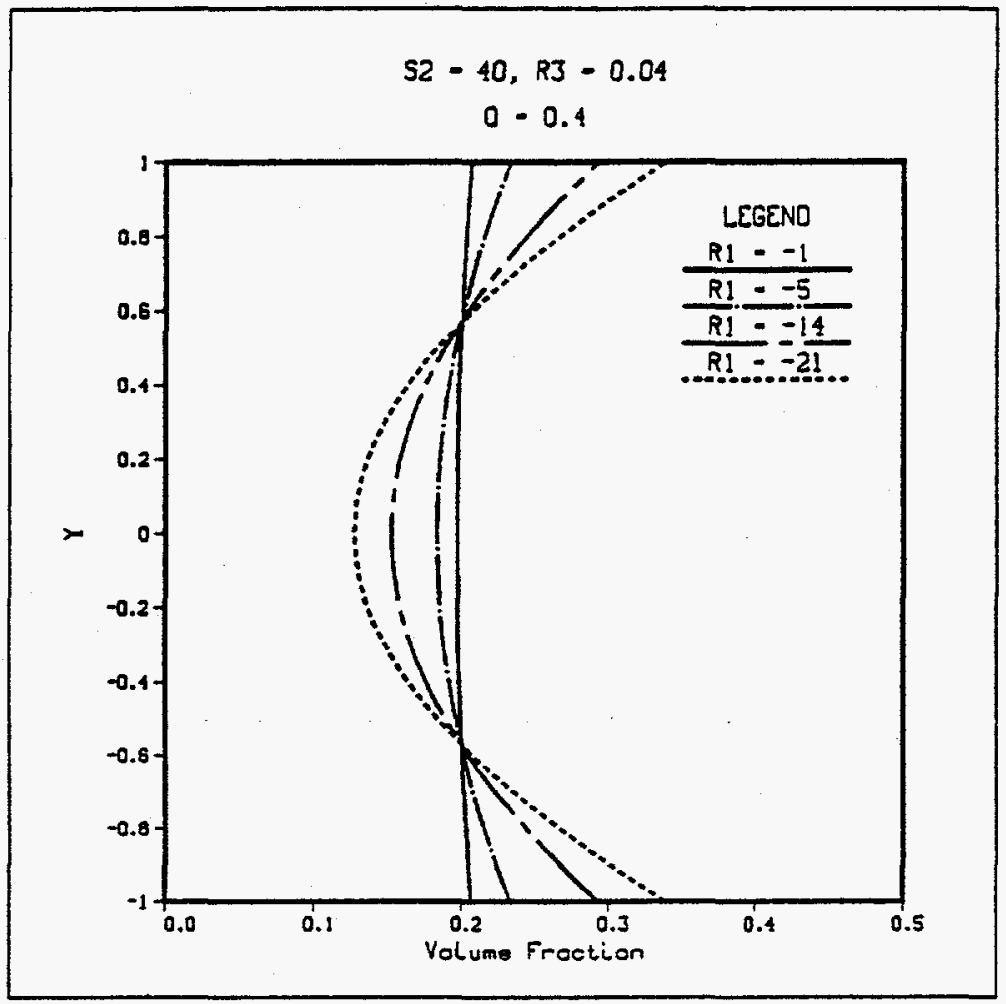

Figure 6. Effect of $R_{1}$ on Volume Fraction Profiles (Case 2).

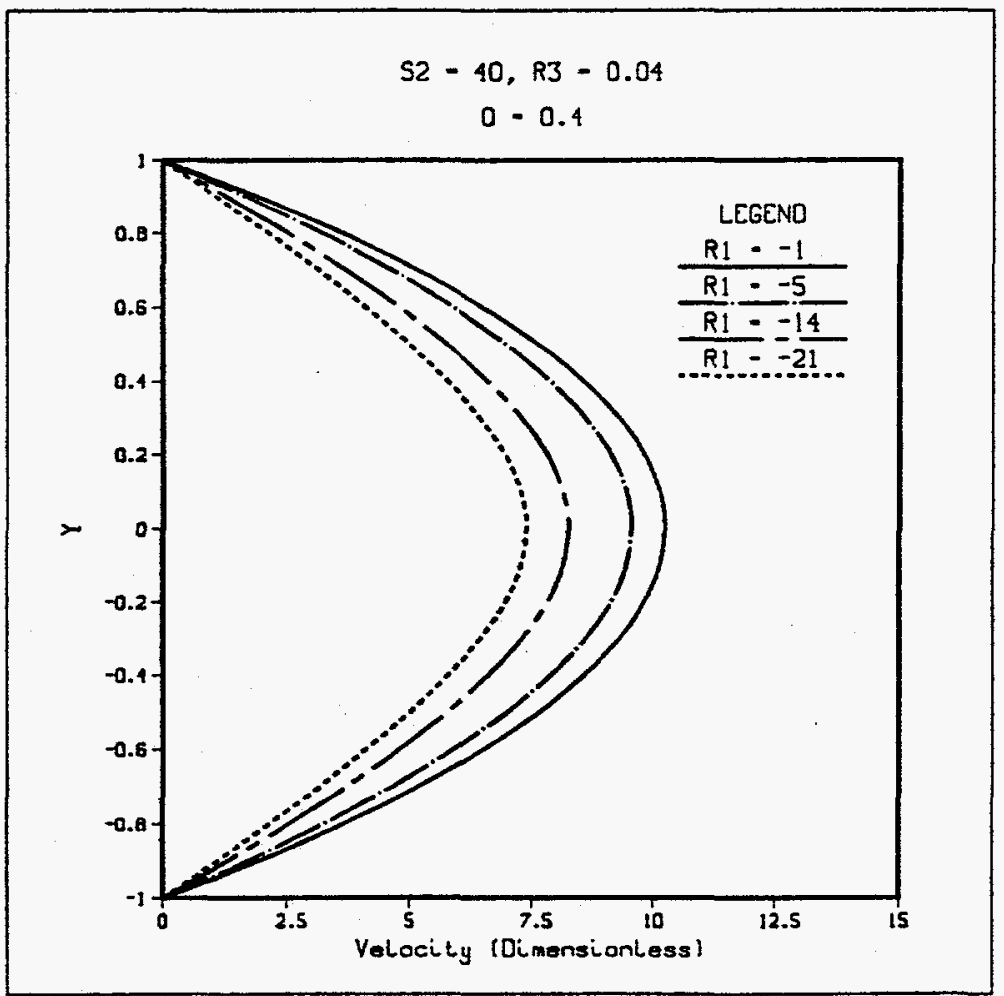

Figure 7. Effect of $R_{1}$ on Velocity Profiles (Case 2). 


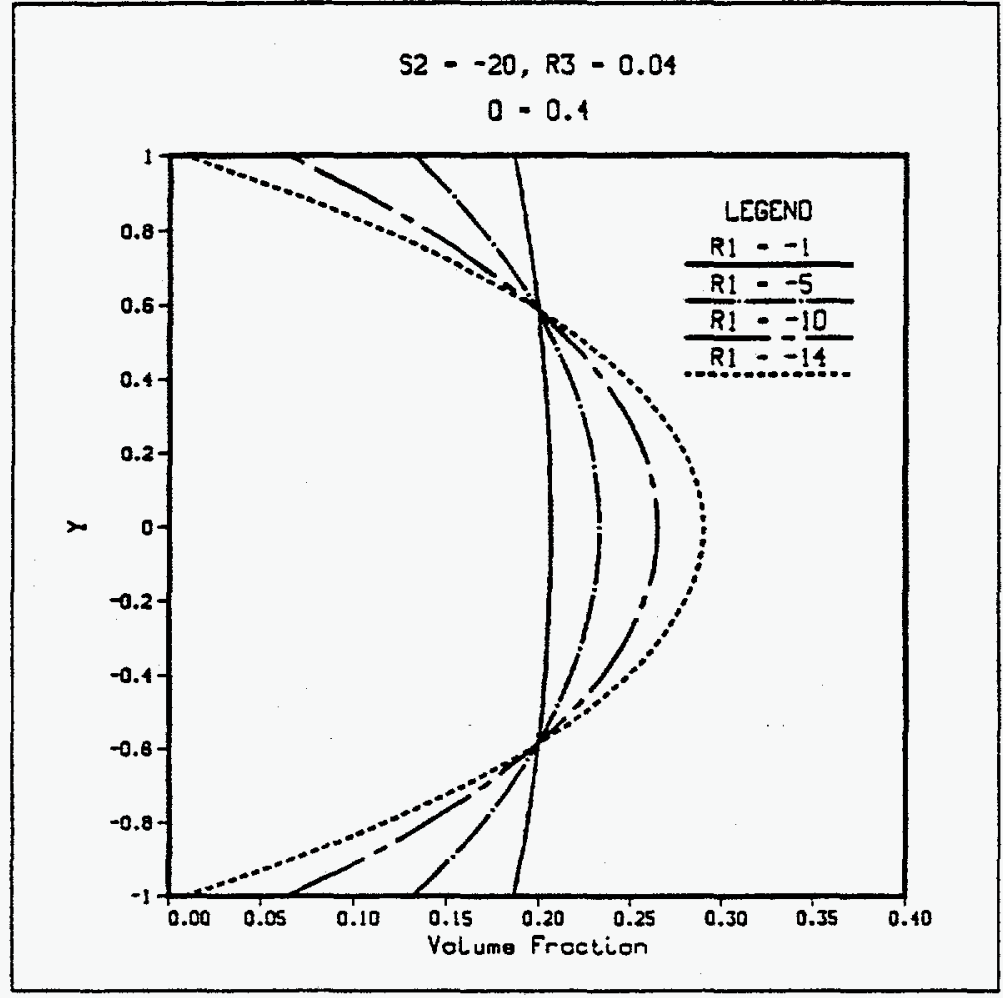

Figure 8. Effect of $R_{1}$ on Volume Fraction Profiles (Case 2).

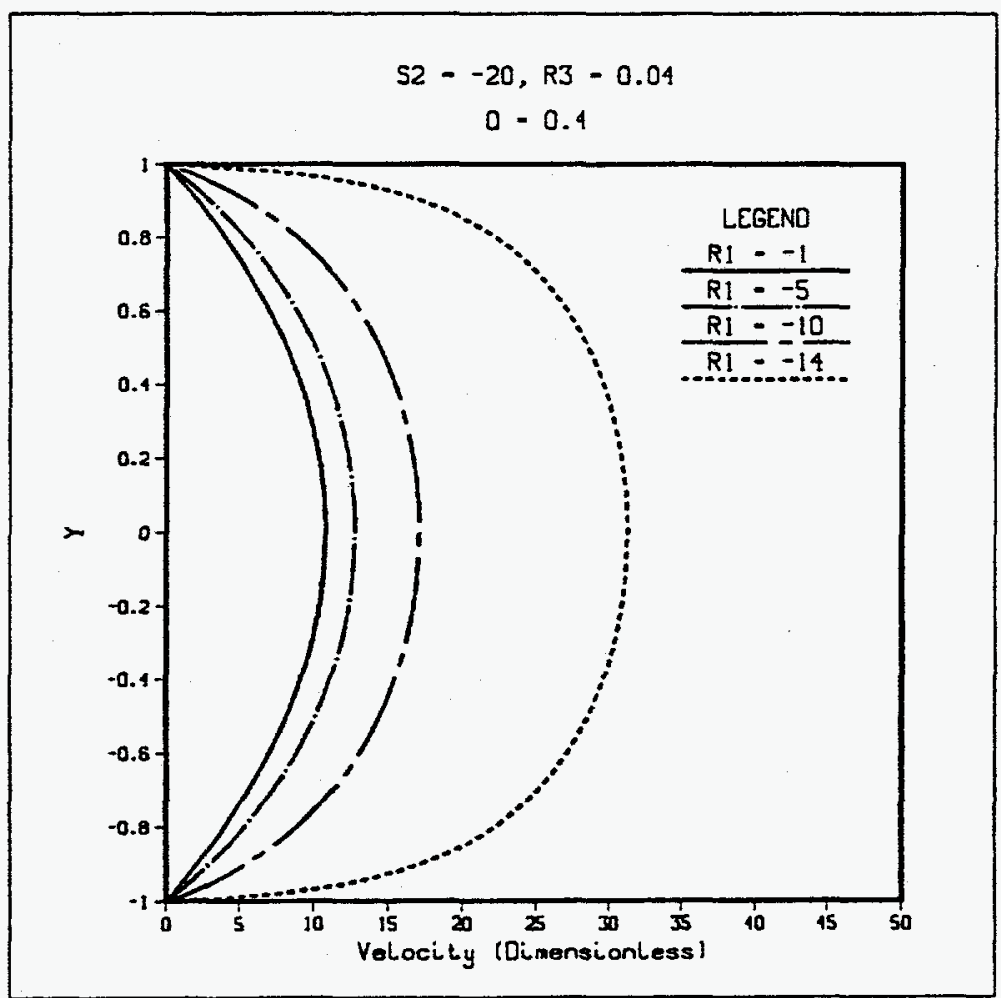

Figure 9. Effect of $R_{1}$ on Velocity Profiles (Case 2). 


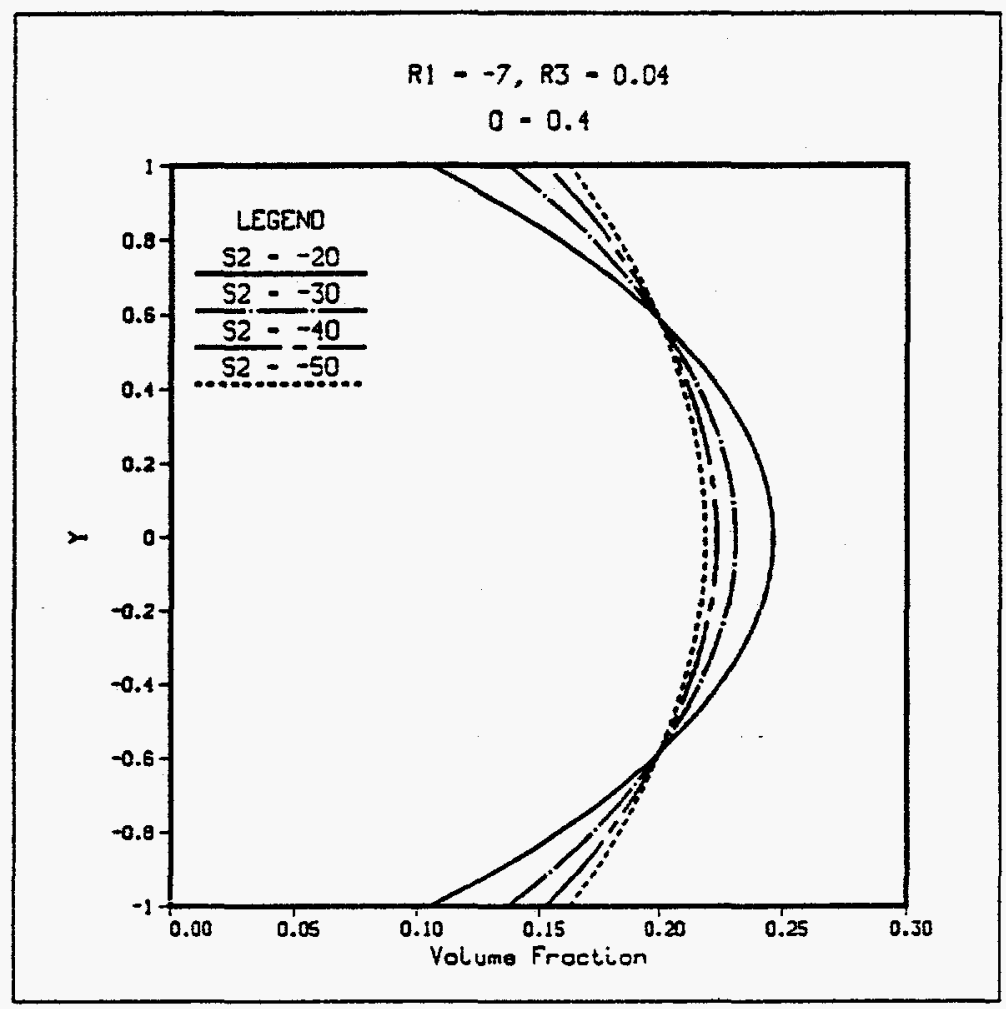

Figure 10. Effect of $S_{2}$ on Volume Fraction Profiles (Case 2).

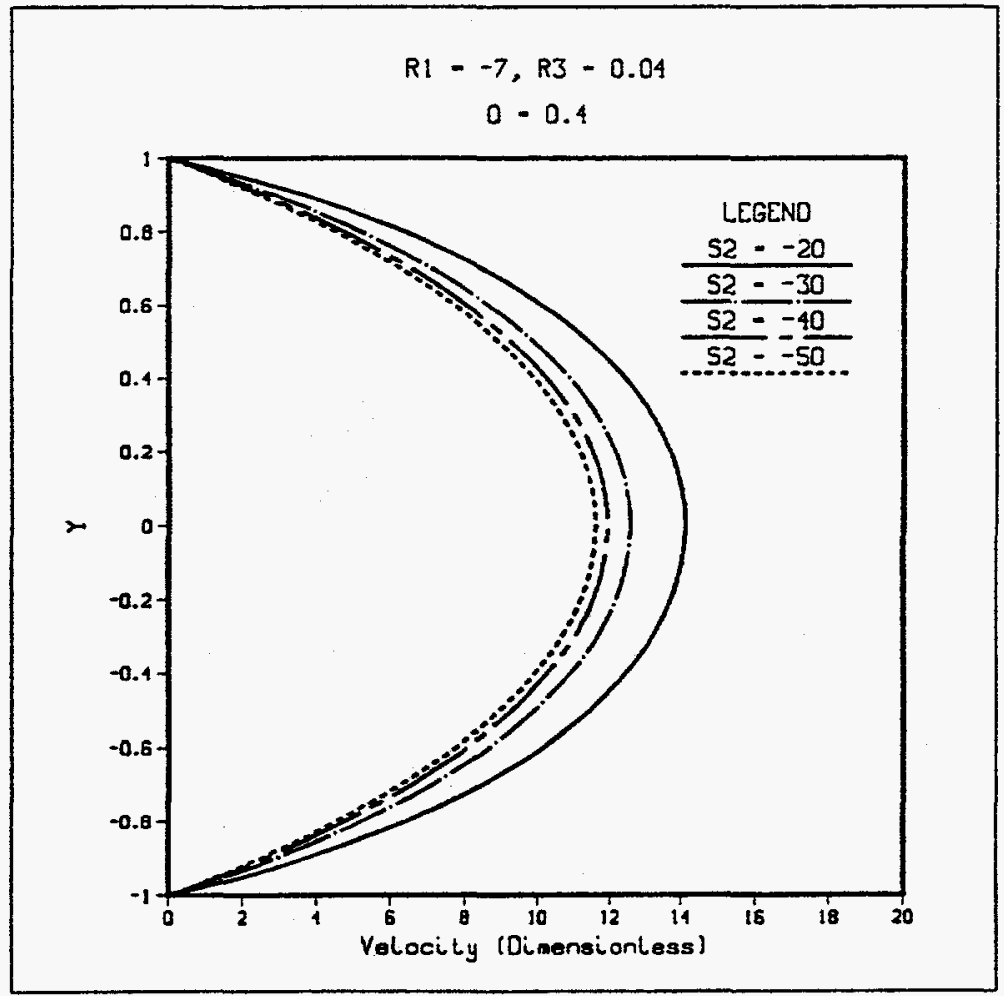

Figure 11. Effect of $S_{2}$ on Velocity Profiles (Case 2). 


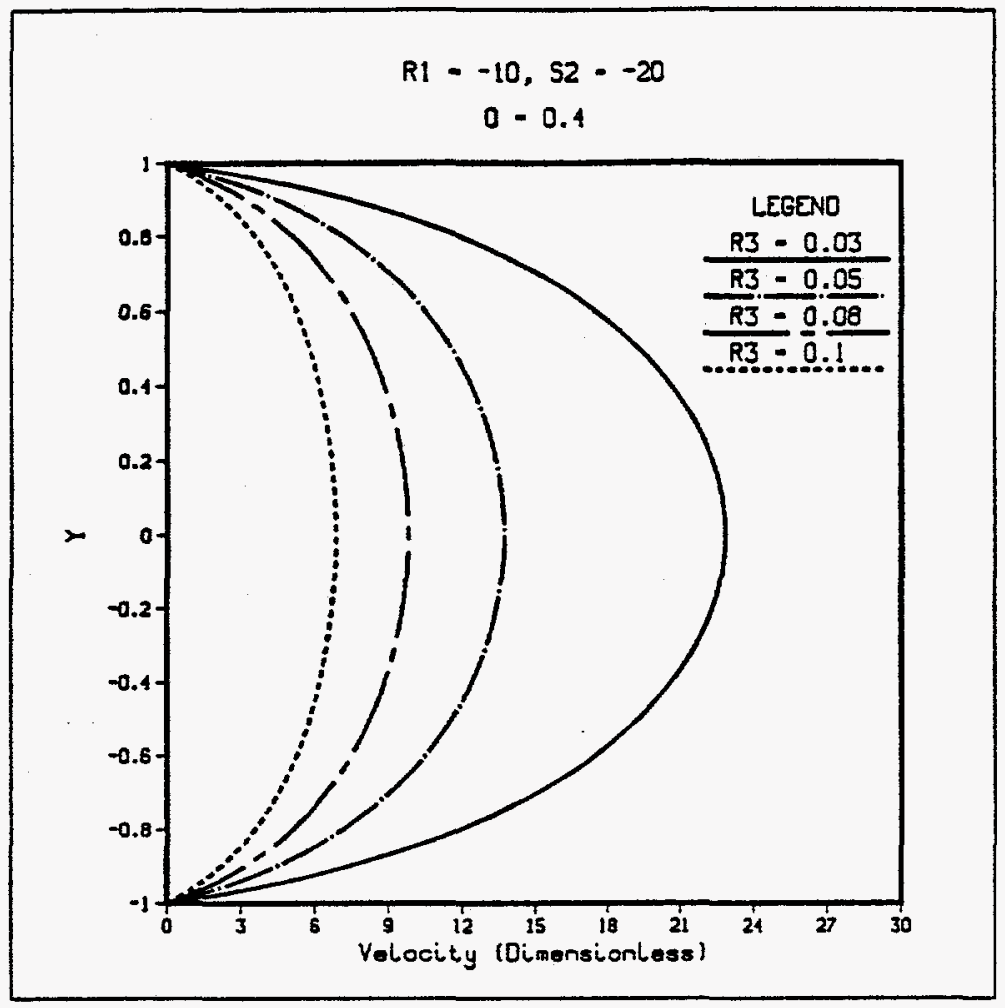

Figure 12. Effect of $R_{3}$ on Velocity Profiles (Case 2).

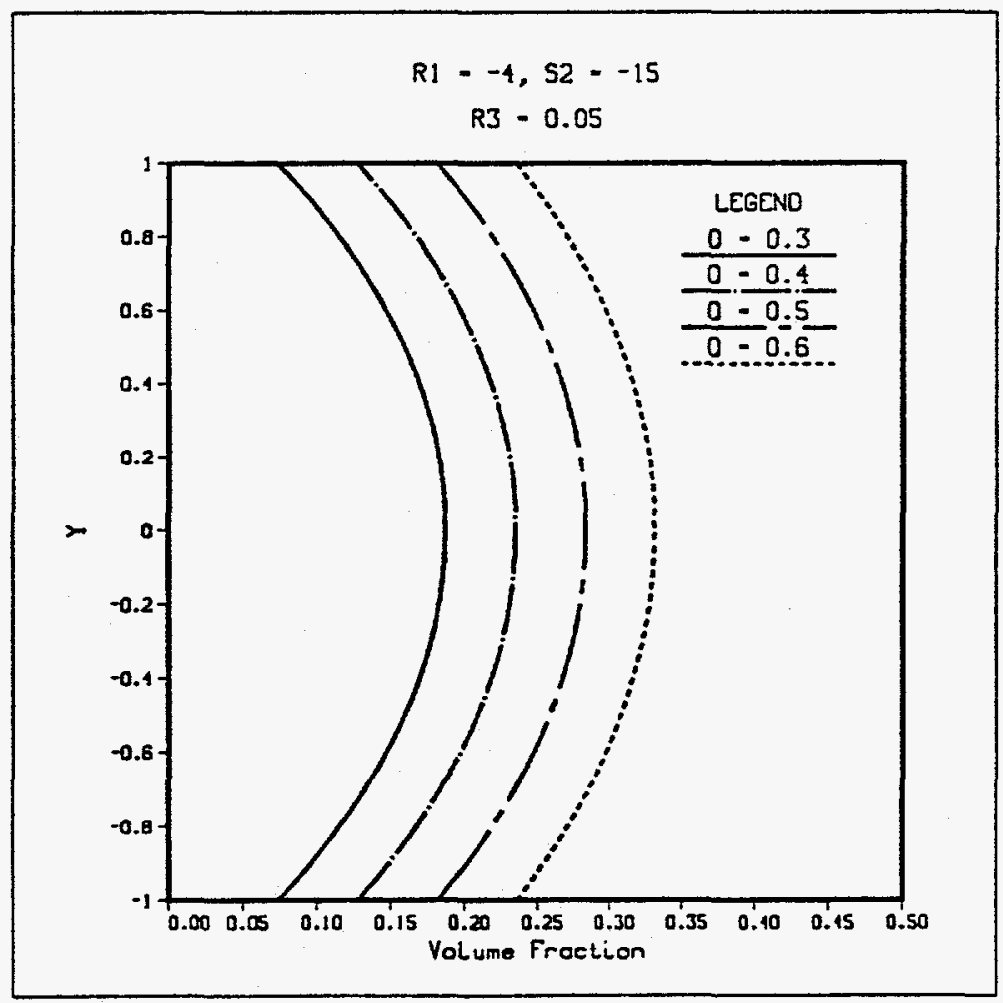

Figure 13. Effect of $Q$ on Volume Fraction Profiles (Case 2). 


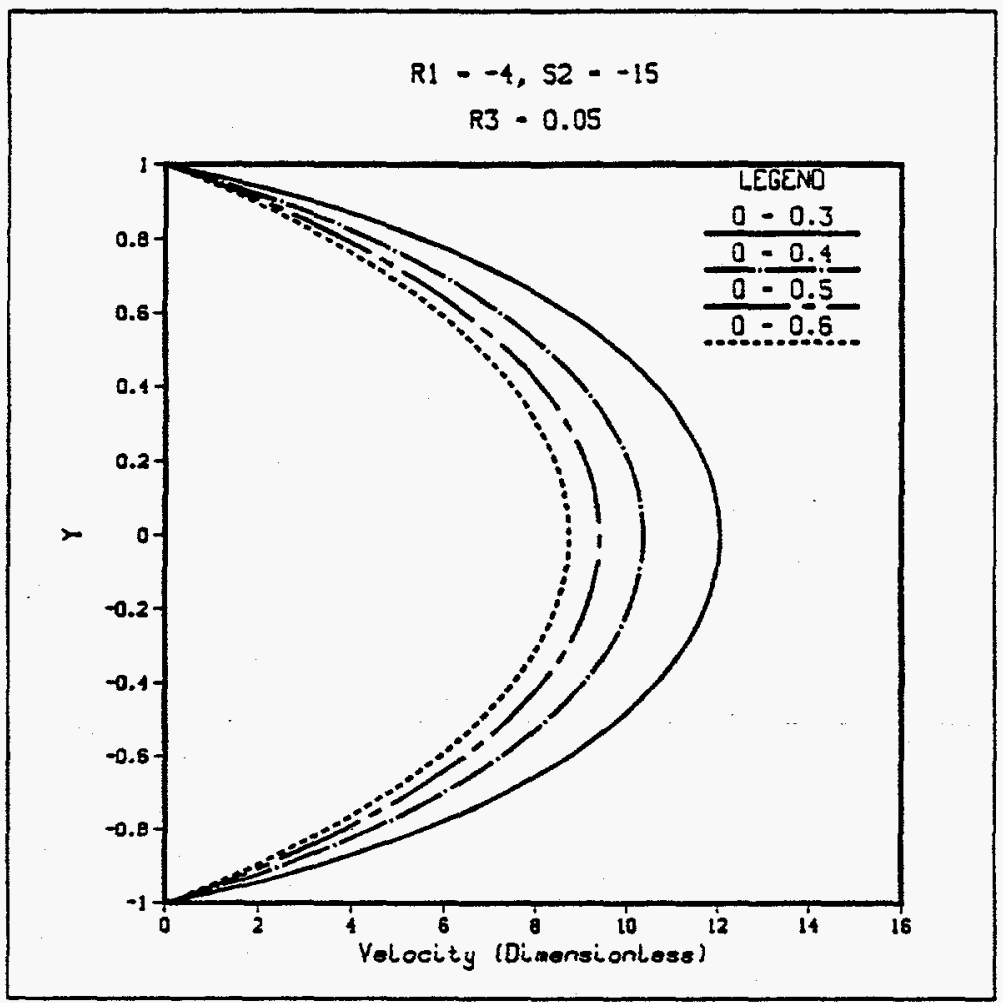

Figure 14. Effect of $Q$ on Velocity Profiles (Case 2).

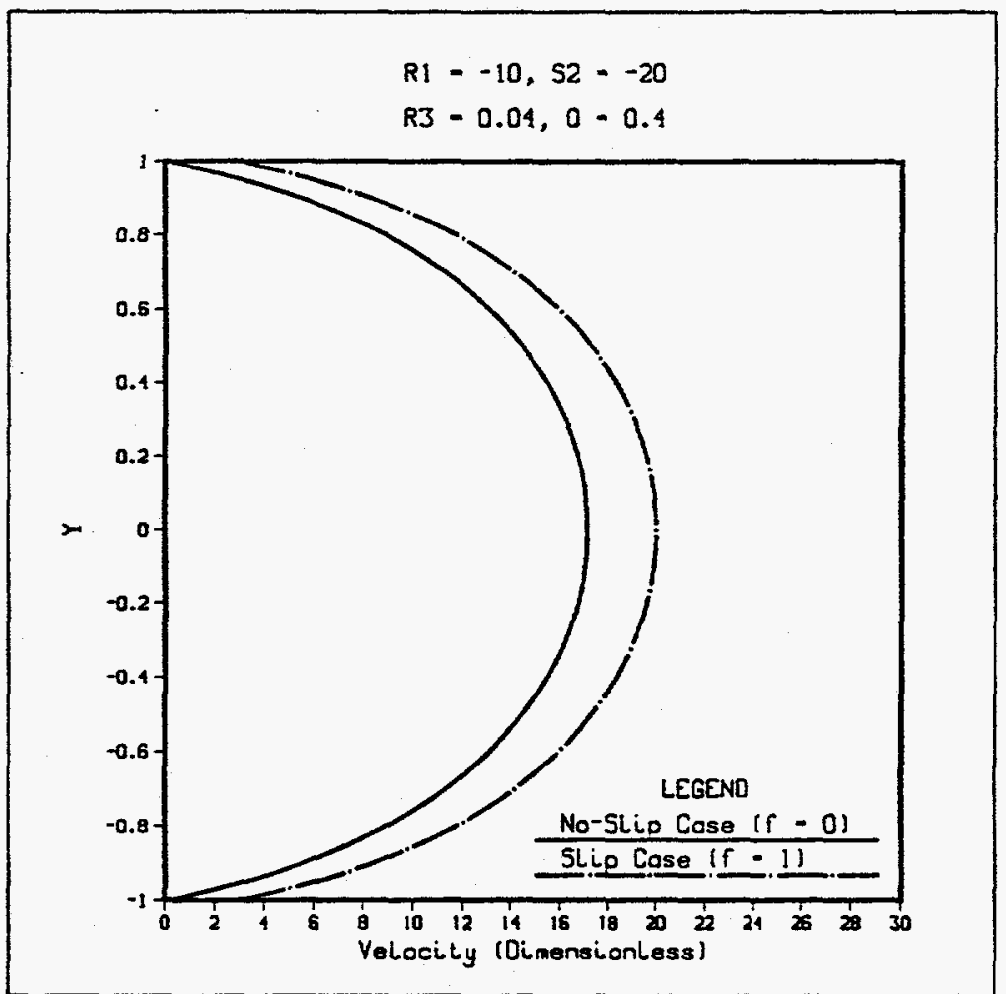

Figure 15. Velocity Profiles for No-Slip and Slip Cases (Case 2). 